\title{
Sex differences in ileal somatostatin-response after stress conditioning in rats
}

\author{
Mari Kimoto ${ }^{1}$, Jorge L. Zeredo ${ }^{3,4}$, Masato S. Ota ${ }^{2}$, Zenro Nihei ${ }^{4}$, Kazuo Toda ${ }^{1,4,}$ * \\ ${ }^{1}$ Physiological Laboratories, Japan Women's University, Tokyo, Japan \\ ${ }^{2}$ Laboratory of Biochemistry and Food Biology, Japan Women's University, Tokyo, Japan \\ ${ }^{3}$ Graduate Program in Health Sciences and Technology, University of Brasilia, Brasilia, Brazil \\ ${ }^{4}$ Integrative Sensory Physiology, Graduate School of Biomedical Sciences, Nagasaki University, Nagasaki, Japan
}

Email address:

k-toda@nagasaki-u.ac.jp (K. Toda)

\section{To cite this article:}

Mari Kimoto, Jorge L. Zeredo, Masato S. Ota, Zenro Nihei, Kazuo Toda. Sex Differences in Ileal Somatostatin-Response after Stress Conditioning in Rats. Journal of Food and Nutrition Sciences. Special Issue: Effects of Foods on Gastrointestinal, Metabolic and Immunological Function. Vol. 3, No. 3-1, 2015, pp. 1-4. doi: 10.11648/j.jfns.s.2015030301.11

\begin{abstract}
We investigated the effects of somatostatin on the ileal movements after gravity stress and compared these effects between male and female rats. Using an in vitro preparation, measurements of ileal movements evoked by somatostatin application were done at 1, 3, 15 and 30 days after $3 \mathrm{G}$ gravity loading-conditioning. Mixed phasic and tonic patterns were observed in the ileal muscle activities. Gravity-stress decreased or antagonized somatostatin inhibitory effects on tonic ileal contraction at an early stage (at day 3 ) in females, but not in males, indicating that sex differences exist in the way that changes of somatostatin sensitivity is modulated by stress conditioning.
\end{abstract}

Keywords: Somatostatin, Gravity Stress, Ileum, Sex Difference, Rat

\section{Introduction}

Stress induces various physiological and hormonal disturbances; in particular, concerning feeding. Previous studies have reported that there is a close relationship between stress and feeding behavior [1-3]. Usually evidenced in both human and animal studies, stress has been shown to affect food intake feeding behavior in a bidirectional fashion, inducing either increases or decreases in food intake [4].

In rats, the severity of the stressor may critically influence the pattern of feeding behavior. For example, chronic and strong stress induces a decrease of in food intake and loss of body weight [5]. By contrast, mild stress increases food intake, especially of highly palatable foods [6,7]. These changes may be in part explained by functional adaptations within the endocrine system [8-10]. For instance, it is generally accepted that corticotropin-releasing hormone $(\mathrm{CRH})$ plays a critical role in the adaptation of the organism to stress. It serves as the main regulating hormone of the hypothalamic-pituitary-adrenal axis, which is activated after exposure to acute stress [11-13]. On the other hand, one of the important gastric hormones to control digestive movements is somatostatin; it inhibits peristalsis in the small intestine of rats and mice. In the small intestine of rats and mice, somatostatin inhibits peristalsis in the small intestine [14], and in the ileum of guinea pigs, it can have both excitatory and inhibitory effects [15-17]. In addition, there are increasing data concerning a functional relationship between stress and somatostatin. It has been found that in the male rat, cold-restraint stress decreases somatostatin sensitivity in the stomach [18], and that ether-stress increases somatostatin release into the pituitary gland [19]. Therefore, it is thought that the effects of stress on digestive function, and by extension on feeding behavior, could take place through a somatostatin-induced functional modulation of peristalsis. Nevertheless, the relationship between stress and somatostatin is still controversial. Further, sex differences are usually observed in stress responses, including changes in digestive function and feeding behavior [20-22].

Here, we investigated the effects of somatostatin on the ileal movements modulated by gravity stress-conditioning and compared these effects between male and female rats.

\section{Methods}

The methods described here follow the ethical guidelines and received approval by the Animal Welfare Committee of 
Japan Women's University.

One hundred and twenty eight rats of both sexes (Wistar, $\mathrm{SPF})$ were divided into Control $(1 \mathrm{G})$ and $3 \mathrm{G}$ groups. Measurements of ileal movements were done at $1,3,15$ and 30 days of gravity-stress conditioning. Each group consisted of 8 rats. Initial body weight (at day 1) was $59.3 \mathrm{~g}$ (mean) in males and $60.0 \mathrm{~g}$ in females and final body weight (at day 30 ) was $267.2 \mathrm{~g}$ in males and $179.2 \mathrm{~g}$ in females, respectively.

\subsection{Ileal Movement Analysis}

For the in vitro preparation, a $1 \mathrm{~cm}$-long portion of the ileum was isolated under barbiturate anesthesia (Nembutal, 20 $\mathrm{mg} / \mathrm{kg}$, i.p.). The inner contents of the isolated ileum were washed away with Tyrode solution. After that, the isolated ileum was fixed into a Magnus-type chamber filled with Tyrode solution (temperature: $37^{\circ} \mathrm{C}$ ) as reported elsewhere [21]. The proximal end of the preparation was set upward and connected to a strain gauge through a cotton thread (Daruma \#30, Yokoi, Osaka, Japan). The distal end of the preparation was fixed to the bottom of the chamber. Ileal movements were amplified by a strain-gauge amplifier (x100) and recorded continuously on a pen-recorder (SS259F2, SEKONIK, Tokyo, Japan).

Ileal movements (spontaneous peristalsis) were observed every $20 \mathrm{~s}$ during $60 \mathrm{~s}$ following the application of somatostatin. Somatostatin solution $(0.3 \mathrm{ml}$ total volume, at the concentration of $10-9 \mathrm{mg} / \mathrm{ml}$ ) was applied topically onto the ileum preparation using an injection syringe. The recovery time after wash-out was too long to do further experiments, thus somatostatin was not tested in other concentrations. Two phases of ileal movements were estimated, that is, the magnitudes of the somatostatin-evoked peak-to-peak amplitudes of the phasic contraction and the tonic amplitudes between baseline and wave peak of the phasic contraction (see Fig.1). Baseline was defined as the bottom line of the phasic deflection just before somatostatin application. The magnitude of the phasic contraction was estimated as the peak-to-peak amplitude of ileal contraction. The magnitude of the tonic contraction was estimated as the baseline-to-peak phasic amplitudes at 20,40 and 60s after somatostatin application.

\subsection{Stress Conditioning}

3G stress was loaded by a centrifugal apparatus (H26-F, Kokusan, Tokyo, Japan) every day at 10:00 AM for $10 \mathrm{~min}$ during 30 days, as described elsewhere [20]. During gravity-loading, rats were placed in the mesh case of the centrifuge with a head-central orientation. In the control group, the rats were placed in the centrifuge for $10 \mathrm{~min}$ but without rotation.

\subsection{Statistics}

A one-way ANOVA with repeated measures was used to determine the differences in tonic ileal contraction between stress and control groups. Where significant differences were found, pairwise comparisons at each time point were done by the post-hoc Bonferroni test. P values lower than 0.05 were considered statistically significant. All data are expressed as means \pm SEM.

\section{Results}

Mixed phasic and tonic patterns were observed in the spontaneous activity of ileal smooth muscles. The former were peristalsis-like movements and the latter presented as a slow negative deflection from the baseline (tonus level) (Fig.1 dotted lines).

Somatostatin induced some irregular rhythms but no change in the average peak-to-peak amplitude of the phasic ileal movements. There were no changes in the phasic responses induced by somatostatin application in relation to stress conditioning.

By contrast, tonic contractions were clearly suppressed by somatostatin application (Fig. 1A). Stress conditioning reversed somatostatin suppression of ileal tonic contraction as compared to control (Fig. 1B). Amplitudes of negative deflection induced by somatostatin application were smaller in female, but not in male rats, after 3 days of stress conditioning. (ANOVA with repeated measures, $F(2,32)=$ $6.038, \mathrm{P}=0.006$ ).

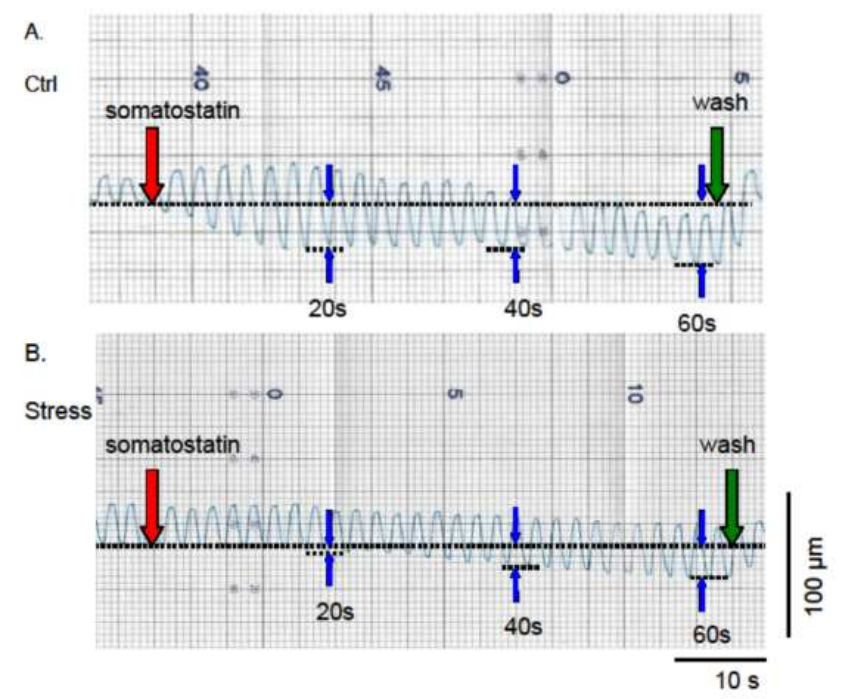

Figure 1. Typical examples of ileum ileal movements and effects of somatostatin application in the female rat. A: Upper trace:Ileal movements in additional gravity $(1 G)$ control rat. B: Lower trace: Ileal movements in $3 G$ stress-conditioned rat. Slow downward movement from the below baseline (dotted line) shows a decrease in tonus. Note that inhibitory effects of somatostatin on the ileal tonus are decreased in $3 G$ gravity stress-conditioned rat as compared to control.

Significant changes were observed from 20 to $60 \mathrm{~s}$ after somatostatin application at day 3 in females (Fig. 2, ANOVA followed by Bonferroni post-hoc test, $\mathrm{P}<0.05$ ). At day 1,15 and 30 , there were no significant differences in the somatostatin effects between control and stress groups both in males and females. 

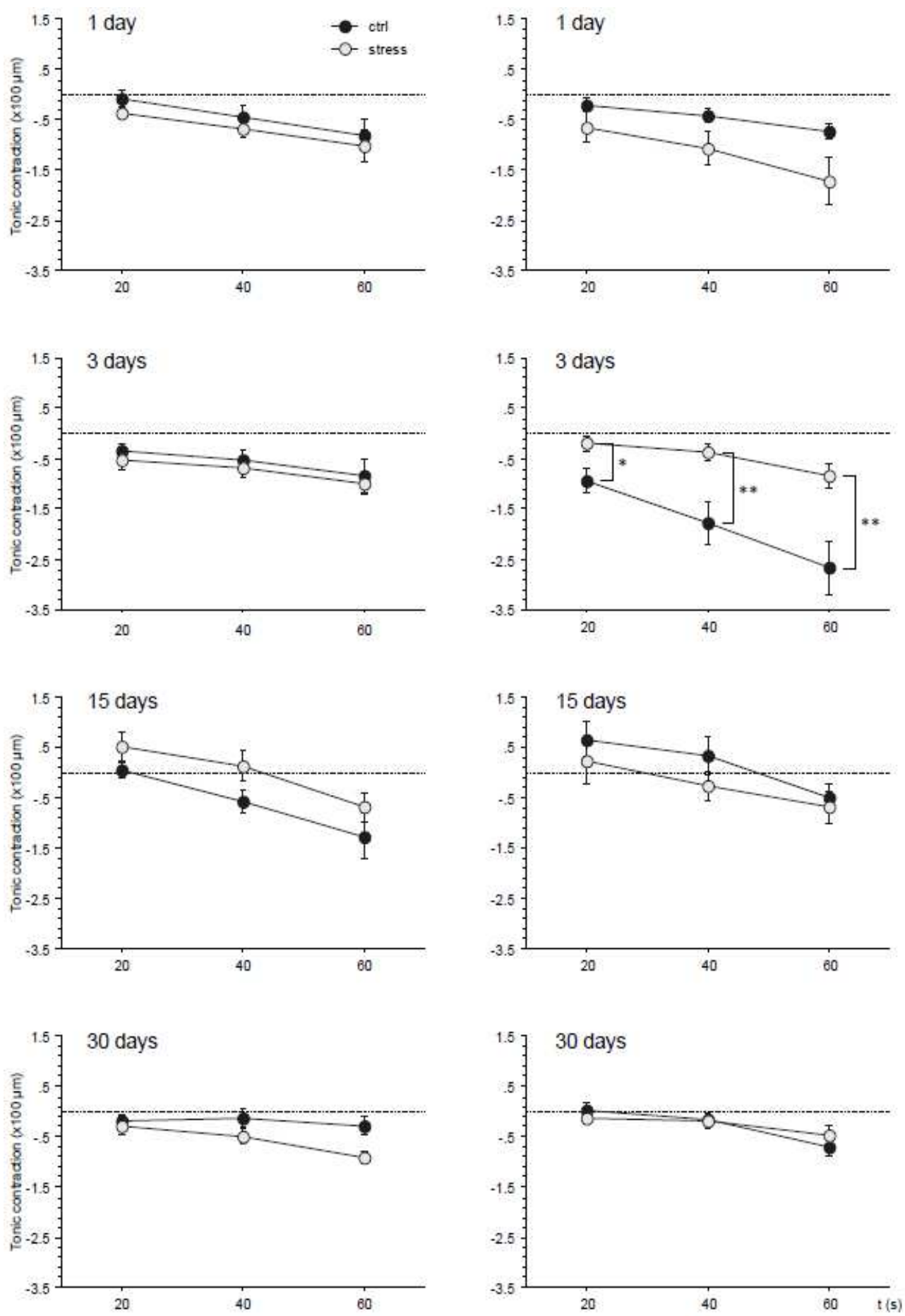

Figure 2. Somatostatin effects on the ileum tonus in males (left-side graphs) and females (right-side graphs) at day 3. Note that at 3 days of stress conditioning, in female rats, the effects of somatostatin were significant antagonized 40-60 s after its application. ${ }^{*} P<0.05, * * P<0.01$, in ANOVA followed by Bonferroni post-hoc test.

\section{Discussion}

Several reports indicate that somatostatin inhibits peristalsis in the rat and mouse jejunum [14,16]. Similarly, in the rat ileum, our present data showed that somatostatin inhibited smooth ileum muscle activities, especially its tonic contraction. In addition, we showed that stress-conditioning decreased or antagonized the somatostatin inhibitory effect on tonic ileal contraction transiently in females at an early stage (at day 3 of conditioning), but not in males. This showed clearly that sex differences exist in the way somatostatin sensitivity can be modulated by gravity-stress. These results suggest that, at least in females, alarm mechanisms against stress were activated either by increasing 
the ileum tonus level or by decreasing the somatostatin sensitivity. In more detail, the fact that stress antagonized the somatostatin-induced inhibition (i.e., disinhibition) on of the tonic ileal contraction suggests that gravity-stress may decrease the somatostatin sensitivity in the female ileum. Because somatostatin release is reported to increase about 2 -fold under stress [19], it is possible that stress conditioning could cause desensitization followed by down-regulation of somatostatin receptors in the ileum.

There are several reports on the sex differences for stress response in the digestive system. In this study, it is not clearly shown why the stress-induced antagonization of the somatostatin effects on the ileal movements was different between males and females. One possible explanation is in the way the endocrine system responds to stress in males and females, which is thought to be critical in determining various physiological functions including digestion $[10,23]$. The present results in relation to somatostatin responsivity expand on previous data that showed sex differences in stress-induced ileal movements $[21,22]$.

In summary, the present study in rats showed that stress reversed the inhibitory effects of somatostatin on the ileal tonus in female, but not in male, suggesting that sex differences exist in the stress-somatostatin interaction in the digestive system. Changes in the sensitivity to somatostatin may partly explain the differences between males and females in the way the digestive system responds to stress.

\section{Acknowledgement}

This study was supported by JSPS grants (20500724 and 25350156, 2008-2014)

\section{References}

[1] Adam ,T.C., Epel, E.S.(2007). Stress, eating and the reward system. Physiol. Behav. 91:449-358.

[2] Patterson, Z.R., Abizaid, A. (2013). Stress induced obesity: lessons from rodent models of stress. Front. Neurosci. 7:130. doi: 10.3389/fnins.2013.00130.

[3] Yau, Y.H., Potenza, M.N. (2013). Stress and eating behaviors.,Minerva Endocrinology 38:255-267.

[4] Torres, S.J., Nowson, C.A. (2007). Relationship between stress, eating behavior, and obesity. Nutrition. 23:887-894.

[5] Marti O, Marti J, Armario A. (1994). Effects of chronic stress on food intakein rats: influence of stressor intensity and duration of daily exposure.Physiol. Behav .55:747-53

[6] Rowland, N.E., Antelman, S.M. (1976). Stress-induced hyperphagia and obesity in rats: a possible model for understanding human obesity. Science191:310-312.

[7] Wallach,M.B., Dawber, M., McMahon,M., Rogers, C. (1977). A new anorexigen assay: stress-induced hyperphagia in rats. Pharmacol. Biochem. Behav. 6:529-531.
[8] Whirledge,S., Cidlowski, J.A.A. (2013). Role for glucocorticoids in stress-impaired reproduction: beyond the hypothalamus and pituitary. Endocrinology. 154:4450-4468.

[9] Machado, T.D., Dalle Molle, R., Laureano, D.P,. Portella, A.K., Werlang, I.C., Benetti C.da, S., Noschang, C., Silveira, P.P. (2013). Early life stress is associated with anxiety, increased stress responsivity and preference for "comfort foods" in adult female rats. Stress 16:549-556.

[10] Lucassen, P.J., Pruessner, J., Sousa, N., Almeida, O.F., Van Dam, A.M., Rajkowska, G., Swaab, D.F., Czéh, B. (2014). Neuropathology of stress. Acta Neuropathol. 127:109-135.

[11] Aguilera. G.(2011). HPA axis responsiveness to stress: implications for healthy aging. Exp. Gerontol. 46:90-95.

[12] Maniam, J., Morris, M.J. (2012). The link between stress and feeding behaviour. Neuropharmacology 63:97-110.

[13] Levy, B.H., Tasker, J.G. (2012). Synaptic regulation of the hypothalamic-pituitary-adrenal axis and its modulation by glucocorticoids and stress. Front Cell Neurosci. 6:24. doi: $10.3389 /$ fncel.2012.00024

[14] Abdu, F., Hicks, G.A., Hennig, G., Allen, J.P,. Grundy, D. (2002). Somatostatin sst(2) receptors inhibit peristalsis in the rat and mouse jejunum. Am. J. Physiol .Gastrointest. Liver Physiol. 282:624-633.

[15] Guillemin, R. (1976). Somatostatin inhibits the release of acetylcholine induced electrically in the myenteric plexus. Endocrinology 99:1653-1654.

[16] Furness, J.B., Costa, M. (1979). Actions of somatostatin on excitatory and inhibitory nerves in the intestine. Eur. J. Pharmacol. 56:69-74.

[17] Feniuk, W., Dimech, J., Humphrey, P.P. (1993). Characterization of somatostatin receptors in guinea-pig isolated ileum, vas deferens and right atrium. Br. J. Pharmacol. 110:1156-1164.

[18] Pizzuto, G., Surgo, D., Clementi, M., Marsico, R., Genco, A., Materia, A., Basso, N. (1997).Differential effect of stress on gastric somatostatin, prostaglandin $\mathrm{E}$ and gastrin release in the rat. Ital J Gastroenterol Hepatol. 29:143-147.

[19] Aguila, M.C., Pickle, R.L.,Yu, W.H., McCann, S.M.(1991). Roles of somatostatin and growth hormone-releasing factor in ether stress inhibition of growth hormone release. Neuroendocrinology 54:515-520.

[20] Kimoto, M., Zeredo, J.L., Toda, K. (2011). Irritant-drinking behaviour can be modified by gravity-stress loaded in developing but not in adult rats. Stress Health 27:34-41.

[21] Kimoto, M., Zeredo, J.L., Toda, K. (2012). Hypergravity conditioning on ileal movements in rats. Aviat. Space Environ. Med. 83:483-487.

[22] Kimoto, M., Zeredo, J.L.,Ota, M.S., Nihei, Z., Toda,K.(2014). Comparison of stress-induced modulation of smooth-muscle activity between ileum and colon in male rats, Auton. Neurosci., 183:8-11.

[23] McEwen, B.S. (2011). Stress, sex and neural adaptation to a changing environment: mechanisms of neuronal remodeling. Ann. N. Y. Acad. Sci. 1204(Suppl): 38-59. 